\title{
Sheaf cohomology in o-minimal structures
}

\author{
Mário J. Edmundo* \\ Gareth O. Jones ${ }^{\dagger}$ \\ CMAF Universidade de Lisboa \\ Department of Mathematics \& Statistics \\ Av. Prof. Gama Pinto 2 \\ McMaster University \\ 1649-003 Lisboa, Portugal \\ 1280 Main Street West \\ Hamilton, Ontario, Canada L8S 4K1 \\ Nicholas J. Peatfield \\ Department of Mathematics \\ University of Bristol \\ Royal Fort Annex \\ Bristol, BS8 1UJ, UK
}

June 30, 2005

\begin{abstract}
Here we prove the existence of sheaf cohomology theory in arbitrary o-minimal structures.
\end{abstract}

\footnotetext{
${ }^{*}$ With partial support from the FCT (Fundação para a Ciência e Tecnologia), program POCTI (Portugal/FEDER-EU) and Fundação Calouste Gulbenkian.

${ }^{\dagger}$ Supported by an EPSRC studentship at University of Oxford.

${ }^{\ddagger}$ Supported by a postdoctoral fellowship from CMAF, Universidade de Lisboa.MSC: 03C64; 55N30. Keywords and phrases: O-minimal structures, sheaf cohomology. Revised on 12th February, 2007.
} 


\section{Introduction}

In the study of groups definable in o-minimal expansions of fields, cohomology has played an important role. For example, in [eo], the torsion of a definably compact, definably connected, definable group is calculated by first computing the cohomology ring of such a group (equipped with its group topology). Using this result, Hrushovski, Peterzil and Pillay [hpp] completed the proof of Pillay's conjecture on recovering Lie groups from definably compact groups. In trying to generalise these results to o-minimal structures not expanding a field, one encountered the problem that cohomological methods were not available. This is because the construction of homology groups (which lead to cohomology groups) by Woerheide [Wo] makes heavy use of triangulation. A consequence of the results we prove in this paper is that for obtaining a cohomology theory, the assumption of a field structure can be weakened to the assumption of a group structure. We show

Theorem 1.1 Suppose that $\mathcal{N}$ is an o-minimal expansion of a group. For a definable set $X$, there exist (functorially associated) cohomology groups $H^{i}(X, \mathbb{Z})$, for $i \geq 0$, which satisfy appropriate versions of the EilenbergSteenrod axioms.

In fact, the coefficients can be taken to be sheaves over a certain site, and the relation between this site and the type space topology from [p] is crucial. Our method for proving the Eilenberg-Steenrod axioms follows the proof by Delfs [D2] in the semialgebraic setting.

Throughout the paper, we work in an arbitrary o-minimal structure $\mathcal{N}=$ $(N,<, \cdots)$. We construct cohomology theories for definable sets, but in order to prove that these satisfy the Eilenberg-Steenrod axioms, we must assume various additional properties. At the end of the paper, to obtain the theorem above, we observe that these properties all hold in expansions of groups.

Acknowledgements. The first author would like to thank Michel Coste, Anand Pillay and Marcus Tressl whose comments during the RAAG School

on O-minimal Structures, June 25-28, 2003, Lisbon, Portugal, were quite helpful for the preparation of this paper. The second author thanks Alex Wilkie for many helpful discussions. We all thank the referee for the much simpler proof of Proposition 2.20 and other useful comments. 


\section{The o-minimal spectrum of definable sets}

Before we start the theory of o-minimal spectra of definable sets, we present the following probably well known result that will be required later.

Proposition 2.1 Every definable set $A \subseteq N^{n}$ is a finite union of definable sets of the form $U \cap F$ where $U$ (resp., $F$ ) is an open (resp., a closed) definable subset of $N^{n}$. Recalling that sets of the form described are called constrcutible this says that every definable set is constructible.

Indeed, by [vdd] page 51, every cell is open in its closure. Hence each cell can be written as $U \cap F$ where $U$ (resp., $F$ ) is an open (resp., a closed) definable subset of $N^{n}$. Thus cell decomposition implies the proposition.

For other basic facts about definable sets and maps we refer the reader to $[\mathrm{vdd}]$.

Definition 2.2 Let $X \subseteq N^{m}$ be a definable set (with parameters in $N$ ). The o-minimal spectrum $\tilde{X}$ of $X$ is the set of complete $m$-types $S_{m}(N)$ of the first-order theory $\operatorname{Th}_{N}(\mathcal{N})$ which imply a formula defining $X$. This is equipped with the topology generated by the basic open sets of the form

$$
\widetilde{U}=\{\alpha \in \widetilde{X}: U \in \alpha\}
$$

where $U$ is a definable, relatively open subset of $X$, and $U \in \alpha$ means the formula defining $U$ is in $\alpha$.

We call this topology on $\widetilde{X}$ the spectral topology.

Observe that the set $\widetilde{X}$ coincides with the set of ultrafilters of the boolean algebra of definable subsets of $X$, and clearly, the sets of the form $\widetilde{U}$ with $U$ open definable subset of $X$ generate a topology on $\widetilde{X}$. In fact, we have $\widetilde{\emptyset}=\emptyset, \widetilde{X}$ is open and $\widetilde{U_{1}} \cap \cdots \cap \widetilde{U_{n}}=\widetilde{U}$ where $U=U_{1} \cap \cdots \cap U_{n}$.

It is immediate that the map $X \longrightarrow \widetilde{X}$, that sends $x \in X$ into the type $\operatorname{tp}(x / N)$ is injective and induces a homeomorphism from $X$, with its strong topology (that is the topology induced on $X$ from the o-minimal product topology on $N^{n}$ ) onto its image in $\widetilde{X}$. Below, we will often identify $X$ with its image in $\widetilde{X}$ under this map.

If $X$ is a definable set, we say that a subset of $\widetilde{X}$ is constructible if it is a finite boolean combination of basic open subsets $\widetilde{U}$. The constructible topology on $\widetilde{X}$ is the topology generated by the constructible subsets of $\widetilde{X}$. Since, by Proposition 2.1, every definable set is a finite boolean combination of open definable sets, it follows that every constructible subset of $\widetilde{X}$ is of 
the form $\widetilde{A}=\{\alpha \in \widetilde{X}: A \in \alpha\}$ where $A$ is a definable subset of $X$ (note that $A$ is not necessarily open, as in the basis for the spectral topology).

Also notice that for any definable $A \subseteq X$ we have that $X \backslash A$ is definable, and so $\widetilde{A}$ is both open and closed in the constructible topology on $\widetilde{X}$. It is a well known model theoretic fact that $\widetilde{X}$ equipped with the constructible topology is a compact, totally disconnected Hausdorff space (see [bs]). In fact, $\widetilde{X}$ with the constructible topology is the Stone space of the boolean algebra of definable subsets of $X$.

Unless otherwise stated, we always consider $\tilde{X}$ equipped with its spectral topology. So when we say "constructible open" we mean open in the spectral topology and constructible, as opposed to "open in the constructible topology".

Remark 2.3 For definable sets $A \subseteq X$, it is easy to see that the following hold:

(1) The tilde operation is an isomorphism from the boolean algebra of definable subsets of $X$ onto the boolean algebra of constructible subsets of $\widetilde{X}$

(2) $A$ is open (resp., closed) if and only if $\widetilde{A}$ is open (resp., closed). Moreover, the tilde operation commutes with the interior and closure operations.

(3) $A$ is definably connected if and only if $\widetilde{A}$ is connected.

We also have the following characterization of open (resp., closed) subsets of $\widetilde{X}$ similar to [BCR] Proposition 7.2.7.

Proposition 2.4 Let $U$ (resp., F) be an open (resp., closed) definable subset of the definable set $X$. Then the following hold:

(1) $\widetilde{U}$ is the largest open subset of $\widetilde{X}$ whose intersection with $X$ is $U$.

(2) $\widetilde{F}$ is the smallest closed subset of $\widetilde{X}$ whose intersection with $X$ is $F$.

Proof. (1) Let $V$ be an open subset of $\widetilde{X}$ such that $V \cap X=U$. Since the constructible open subsets form a basis of the topology of $\widetilde{X}$, it follows that $V=\cup\{\widetilde{B}: B$ is an open definable subset of $X$ such that $\widetilde{B} \subseteq V\}$. But if $\widetilde{B} \subseteq V$, then $B=\widetilde{B} \cap X \subseteq U$, and, hence, $\widetilde{B} \subseteq \widetilde{U}$. Thus $V \subseteq \widetilde{\widetilde{U}}$.

(2) is obtained from (1) by taking complements.

The next result is easy and is from $[\mathrm{p}]$. Recall that a set $X$ in a topological space is said to be irreducible if and only if it is not the union of any two proper closed subsets. 
Proposition 2.5 Let $X$ be a definable set. The space $\widetilde{X}$ is $T_{0}$, quasi-compact and a spectral space, i.e.: (i) it has a basis of quasi-compact open subsets, closed under taking finite intersections; and (ii) each irreducible closed subset is the closure of a unique point.

Proof. First we show that $\tilde{X}$ is $T_{0}$, so suppose $\alpha \neq \beta \in \widetilde{X}$. Since we can consider them as distinct complete types there must be a formula defining an open subset, $U$, of $X$ which is in, without loss, $\alpha$ and not in $\beta$. If there were no such $U$ then $\alpha$ and $\beta$ contain all the same open sets, and hence all the same closed sets. But then, since by Proposition 2.1, every definable set is constructible, they contain all the same definable sets, and so are the same. The open set $\widetilde{U}=\{\gamma \in \widetilde{X}: U \in \gamma\}$ contains $\alpha$ and not $\beta$.

The basic open subsets $\widetilde{U}$ and $\widetilde{X}$ itself, are quasi-compact since the constructible topology is finer than the spectral topology.

Now let $F \subseteq \widetilde{X}$ be closed and irreducible. Let $\Phi=\{B \subseteq X: B$ is closed, definable and $B \in \beta$ for all $\beta \in F\}$. Let $\Psi=\Phi \cup\{X \backslash C: C$ is closed, definable and $C \notin \Phi\}$. By irreducibility of $F, \Psi$ is consistent and thus determines a type $\gamma \in \widetilde{X}$. Clearly, $F$ is the closure of $\gamma$ and only of $\gamma$.

Note that Hochster shows in $[\mathrm{h}]$ that the spectral spaces are exactly the spaces homeomorphic to the prime spectrum of a (commutative) ring with identity element, equipped with the Zariski topology.

Definition 2.6 Let $X$ be a definable set and $\alpha, \beta \in \widetilde{X}$. We say that $\beta$ is a specialization of $\alpha$ (or $\alpha$ is a generalization of $\beta$ ) if and only if $\beta$ is in the closure of $\{\alpha\}$.

The notion of specialization is valid for any spectral space and defines a partial order on the set of points. The following property holds in any spectral space (compare with [BCR] Proposition 7.1.21).

Proposition 2.7 Let $X$ be a definable set and $C$ a constructible subset of $\widetilde{X}$. Then $C$ is closed (resp., open) in $\widetilde{X}$ if and only if it is stable under specialization (resp., generalization) in $\widetilde{X}$.

We now investigate if as in the real algebraic case in [BCR] Chapter 7 the specializations of a point in the o-minimal spectrum of a definable set form a chain. The proof in $[\mathrm{BCR}]$ is based on real algebra and does not work in the o-minimal context. However we have the following remark. 
Remark 2.8 O-minimality implies that for any complete 1-type, $\alpha$, over $N$ we only have the following possibilities:

- $\alpha$ is the type of a point $a \in N$, in which case we often abuse notation and write $a$ for it's type;

- the type of $+\infty$ or $-\infty$, when we similarly abuse notation;

- the type of an element transcendental over $N$ which defines a Dedekind cut in it's ordering (transcendental in the model theoretic sense, that is the type of an element which is not definable over $N$ );

- $\alpha$ is the type of an element infinitesimally above (or below) $a \in N$ i.e. the type containing all the open intervals $(a, b)$ (resp. $(b, a))$ for all $b>a($ resp. $b<a)$, when we say $\alpha=a^{+}\left(\right.$resp. $\left.a^{-}\right)$;

We get the last two possibilities from the case that $\alpha$ contains some bounded interval, say $(a, b)$. For every such open interval in $\alpha$ and every $c \in(a, b)$, either $(a, c)$ or $(c, b)$ is in $\alpha$, since it is complete. If all the subintervals of $(a, b)$ in $\alpha$ are of the form $(a, c)$ (resp. $(c, b))$ then we have that $\alpha=a^{+}$(resp. $\left.b^{-}\right)$. If there is no open interval $(a, b)$ in $\alpha$ such that either $(a, c) \in \alpha$ for all $c>a$ or $(c, b) \in \alpha$ for all $c<b$, then $\alpha$ defines a cut at a transcendental element by the pair of sets: $\{x$ : there is $(a, b) \in \alpha$ with $a>x\}$ and $\{x$ : there is $(a, b) \in \alpha$ with $b<x\}$. Clearly the cut has to be at a transcendental element or we could define $\alpha$ to be a point definable over $N$.

Only types of the final kind are not closed points of $\widetilde{X}$. It can easily be checked that $a \in N$ is a specialisation of $a^{+}$and $a^{-}$but not vice-versa.

This also gives that the sets $\{a\}, \overline{\left\{a^{+}\right\}}=\left\{a, a^{+}\right\}$and $\overline{\left\{a^{-}\right\}}=\left\{a, a^{+}\right\}$are irreducible, so the set $\left\{a^{-}, a, a^{+}\right\}$is not.

The following example shows that without some assumptions on the structure $\mathcal{N}$, we do not have that the specializations of $\alpha \in \widetilde{X}$ form a chain if $X \subseteq N^{m}$ and $m>1$.

Example 2.9 Let $\mathcal{N}=(\mathbb{Q},<)$ and take $X=\mathbb{Q}^{2}$. For any $a<b \in \mathbb{Q}$ let $\alpha$ be the type given by the ordered pair $\left\langle a^{-}, b^{-}\right\rangle$, that is the type of an infinitesimal box below and to the left of the point $\langle a, b\rangle$. Then let $\beta$ be the type given by the ordered pair $\left\langle a^{-}, b\right\rangle$ and $\gamma$ be the type given by the ordered pair $\left\langle a, b^{-}\right\rangle$. Then $\beta$ and $\gamma$ are specializations of $\alpha$ since basic the open sets in $\beta$ (respectively $\gamma$ ) are all of the form $(c, d) \times(e, f)$ for $c<a \leq d$ and $e<b<f$ (respectively $(c, d) \times(e, f)$ for $c<a<d$ and $e<b \leq f$ ) and all of these sets are also in $\alpha$. But neither $\beta$ nor $\gamma$ is a specialization of the other, since $(c, a) \times(e, f)$ is in $\beta$ but not $\gamma$ and $(c, d) \times(e, b)$ is in $\gamma$ but not $\beta$. 
We now present an example to show that the closed specialisation of $\alpha \in \widetilde{X}$ is not necessarily unique without further assumptions on $X$. Thanks to Alf Onshuus for bringing it to our attention.

Example 2.10 We take $\mathcal{N}$ as in Example 2.9 and $X=\left\{\langle x, y\rangle \in \mathbb{Q}^{2}\right.$ : $\langle x, y\rangle \neq\langle a, b\rangle\}$ for some fixed $a$ and $b$ in $\mathbb{Q}$ with $a<b$. Again letting $\alpha=$ $\left\langle a^{-}, b^{-}\right\rangle \in \widetilde{X}$ we get that the closure of $\{\alpha\}$ in $\widetilde{\mathbb{Q}^{2}}$ is the set $\left\{\alpha,\left\langle a, b^{-}\right\rangle,\left\langle a^{-}, b\right\rangle\right.$, $\langle a, b\rangle\}$, with $\langle a, b\rangle$ being the only point closed in $\widetilde{\mathbb{Q}^{2}}$. But in $\widetilde{X}$ we do not have this point, and so both $\left\langle a^{-}, b\right\rangle$ and $\left\langle a, b^{-}\right\rangle$are closed points of $\widetilde{X}$ which are specialisations of $\alpha$.

There is a result from the second authors D.Phil. thesis showing that if $\mathcal{N}$ is an o-minimal expansion of a real closed field and $X$ is a definable set, then the specializations of every $\alpha \in \widetilde{X}$ form a chain and $\alpha$ is the generalization of a unique closed point. Our next goal is to find weaker conditions on a definable set $X$ in an arbitrary o-minimal structure $\mathcal{N}$ such that each point of $\widetilde{X}$ is the generalisation of unique closed point.

Lemma 2.11 Given a definable set $X \subseteq N^{m}$ and $\alpha \in \tilde{X}$ there is some closed type (i.e. a closed point in the spectral topology), $\beta$, which is a specialisation of $\alpha$, and for any $A \in \alpha \backslash \beta$ we have $\operatorname{fr}(A) \in \beta$ where $\operatorname{fr}(A)=\bar{A} \backslash A$ is the frontier of $A$.

Proof. We go by induction on $\operatorname{dim}(\alpha):=\min \{\operatorname{dim}(A): A \in \alpha\}$. If $\operatorname{dim}(\alpha)=$ 1 then $\alpha$ contains a one dimensional set $A$, which, by cell-decomposition and the completeness of $\alpha$, we can assume is a cell. By o-minimality we have that $A$ can be definably totally ordered, and thus that any type containing $A$ is one of those described in Remark 2.8. Thus $\alpha$ is either closed (in which case it is clearly it's own unique closed specialisation) or $\alpha=a^{+}$or $a^{-}$, for $a \in A \subseteq X$ in which case the unique close specialisation of $\alpha$ is $a$.

For $\operatorname{dim}(\alpha)>1$, first note that if $\alpha$ is closed then by the same reason as above we are done, so assume $\alpha$ not closed. Thus we can find a specialisation $\beta$ of $\alpha$ distinct from $\alpha$. Then we take any $A^{\prime} \in \alpha \backslash \beta$ and any $A^{\prime \prime} \in \alpha$ realising $\operatorname{dim}(\alpha)$ and let $A=A^{\prime} \cap A^{\prime \prime}$.

If $\operatorname{fr}(A)$ is not in $\beta$ then $N^{k} \backslash \operatorname{fr}(A)=\left(N^{k} \backslash \bar{A}\right) \cup A \in \beta$ and since we already have $N^{k} \backslash A \in \beta$ we have that their intersection, $N^{k} \backslash \bar{A} \in \beta$. But this is open, and so, as $\beta$ is a specialisation of $\alpha$, we must also have $N^{k} \backslash \bar{A} \in \alpha$. But since $A \in \alpha$ this contradicts the consistency of $\alpha$.

So $\operatorname{fr}(A) \in \beta$, and since $\operatorname{dim}(\operatorname{fr}(A))<\operatorname{dim}(A)$ we must have $\operatorname{dim}(\beta)<$ $\operatorname{dim}(\alpha)$. By the induction hypothesis $\beta$ has a closed specialisation, which is thus also a closed specialisation of $\alpha$, and we are done. 
Proposition 2.12 A spectral space is normal if and only if every point has a unique closed specialization.

Proof. This is part of Proposition 2 of [cc].

Theorem 2.13 Given definable $X \subseteq N^{k}$ then the following are equivalent:

(1) $\tilde{X}$ is normal. In fact, if $F$ and $G$ are two disjoint closed subsets of $\widetilde{X}$ then there exist two disjoint constructible open (i.e. open in the spectral topology and constructible) subsets $U$ and $V$ of $\widetilde{X}$ such that $F \subseteq U$ and $G \subseteq V$.

(2) $X$ is definably normal (i.e. for disjoint definable closed sets $F, G$ in $X$ there are disjoint definable open sets $U$ and $V$ such that $F \subseteq U$ and $G \subseteq V)$.

Proof. That (1) implies (2) follows from the fact that any sets $F$ and $G$ as in the statement of (2) give rise to disjoint $\widetilde{F}$ and $\widetilde{G}$ closed in the spectral topology. By (1) these can be separated by constructible open sets $\widetilde{U}$ and $\widetilde{V}$ which, by their constructibility, come from open sets $U$ and $V$ which separate $F$ and $G$ in $X$. This is what we need.

Now let (3) be the statement that any point $\alpha \in \widetilde{X}$ has a unique closed specialization. Then Proposition 2.12 gives that (3) implies (1). We prove that (2) implies (3) which gives the result. In fact we show that (1), (2) and (3) are equivalent by induction on $\operatorname{dim}(X)$. Let $\left(1_{\mathrm{m}}\right),\left(2_{\mathrm{m}}\right),\left(3_{\mathrm{m}}\right)$ be the statements restricted to $X$ of dimension $m$. By the above we have $\left(3_{\mathrm{k}}\right) \Longrightarrow\left(1_{\mathrm{k}}\right)$ and $\left(1_{\mathrm{k}}\right) \Longrightarrow\left(2_{\mathrm{k}}\right)$ for all $k$. We show now that if $\left(1_{\mathrm{k}}\right),\left(2_{\mathrm{k}}\right),\left(3_{\mathrm{k}}\right)$ are equivalent for all $k \leq m$ and $\left(2_{\mathrm{m}+1}\right)$ holds, then $\left(3_{\mathrm{m}+1}\right)$ holds, which will complete the proof.

We first show that $\left(3_{1}\right)$ (and therefore $\left(1_{1}\right)$ and $\left(2_{1}\right)$ ) hold in any case. If $\operatorname{dim}(X)=1$ then by cell-decomposition $X$ is a finite union of disjoint 1 and 0 dimensional sets, $U_{i}$, each of which is definably totally ordered. For any $\alpha \in \widetilde{X}$ is in some unique $\widetilde{U}_{i}$. By o-minimality, types in any of the $\widetilde{U}_{i}$ are given in the same way as the types in some $\widetilde{Y}$ for $Y \subseteq N$, with respect to this new order. By Remark 2.8 any such type is either closed or equals $a^{+}$ or $a^{-}$for some $a \in Y$, and thus has unique closed specialisation $a$. In either case the type has a unique closed specialisation. This proves $\left(3_{1}\right)$.

We now show that $\left(1_{\mathrm{k}}\right) \Longrightarrow\left(1_{\mathrm{k}}^{\prime}\right)$ for all $k$, where $\left(1_{\mathrm{k}}^{\prime}\right)$ is the statement: 
$\left(1_{\mathrm{k}}^{\prime}\right)$ Given $\beta \in O \subseteq \widetilde{X}$, where $\operatorname{dim}(X)=k, \beta$ is a closed point and $O$ is a constructible open set, there is a constructible open $W_{\beta}$ set such that $\beta \in W_{\beta} \subseteq \overline{W_{\beta}} \subseteq O$.

We use $\left(1_{\mathrm{k}}\right)$ with $F=\{\beta\}$ and $G=\widetilde{X} \backslash O$ to get constructible open $U$ and $V$ such that $\beta \in U$ and $\widetilde{X} \backslash O \subseteq V$ and $U \cap V=\emptyset$. Putting $W_{\beta}=U$ we get the result, since $\bar{U} \subseteq \widetilde{X} \backslash V$, as the set on the right is closed and contains $U$, and also $\widetilde{X} \backslash V \subseteq O$, since $\widetilde{X} \backslash O \subseteq V$.

Assume that $\left(3_{\mathrm{k}}\right),\left(1_{\mathrm{k}}\right),\left(2_{\mathrm{k}}\right)$ are equivalent for all $k \leq m$ and $X$ is a definably normal definable set with $\operatorname{dim}(X)=m+1$. Let $\alpha \in \widetilde{X}$ and take two distinct closed types, $\beta$ and $\gamma$, which are both specialisations of $\alpha$. Take any $A^{\prime} \in \alpha$ such that $\operatorname{dim}\left(A^{\prime}\right)=\operatorname{dim}(\alpha)$, any $B \in \alpha \backslash \beta$ and any $C \in \alpha \backslash \gamma$. Then let $A$ be a cell in $A^{\prime} \cap B \cap C$ which is in $\alpha$ (there is such a thing, by cell-decomposition, because $\alpha$ is consistent and complete). Then $\operatorname{dim}(A)=\operatorname{dim}(\alpha), A \in \alpha \backslash \beta, A \in \alpha \backslash \gamma$ and $\operatorname{fr}(A)$ is closed (as $A$ is a cell and cells are open in their closures) of dimension $k$ strictly less than $\operatorname{dim}(A)$, and hence less than or equal to $m$. Also by Lemma 2.11 we have $\operatorname{fr}(A) \in \beta$ and $\operatorname{fr}(A) \in \gamma$, so $\beta, \gamma \in \widetilde{\operatorname{fr}(A)}$.

A closed definable subset of a definably normal set is definably normal, so since $\operatorname{fr}(A)$ is a closed definable subset of $X$ and $X$ is definably normal, $\operatorname{fr}(A)$ is definably normal, i.e., $\left(2_{\mathrm{k}}\right)$ holds for $\operatorname{fr}(A)$ in place of $X$. Thus we can use $\left(1_{\mathrm{k}}\right)$ to get disjoint $U_{\beta} \ni \beta$ and $U_{\gamma} \ni \gamma$ constructible open in $\widetilde{\operatorname{fr}(A)}$. Then by $\left(1_{\mathrm{k}}^{\prime}\right)$ we get $W_{\beta}, W_{\gamma}$ constructible open in $\widetilde{\operatorname{fr}(A)}$ such that $\beta \in W_{\beta} \subseteq \overline{W_{\beta}} \subseteq U_{\beta}$, and similarly for $\gamma$. As $W_{\beta}, W_{\gamma}$ are constructible, by Remark 2.3 (1), we have that there are definable $C_{\beta}$ and $C_{\gamma}$ in $X$ such that $\overline{W_{\beta}}=\widetilde{C_{\beta}}$ and $\overline{W_{\gamma}}=\widetilde{C_{\gamma}}$, with $C_{\beta}$ and $C_{\gamma}$ closed in $\operatorname{fr}(A)$, and hence closed in $X$. So by definable normality of $X,\left(2_{\mathrm{m}+1}\right)$, there are disjoint definable sets $V_{\beta}$ and $V_{\gamma}$ open in $X$ such that $C_{\beta} \subseteq V_{\beta}$ and $C_{\gamma} \subseteq V_{\gamma}$. Then $\widetilde{V_{\beta}}$ and $\widetilde{V_{\gamma}}$ are disjoint and $\beta \in \widetilde{C_{\beta}} \subseteq \widetilde{V_{\beta}}$. But as $\beta$ is a specialisation of $\alpha$ this gives $\alpha \in \widetilde{V_{\beta}}$, and arguing similarly with $\gamma$ in place of $\beta$ gives $\alpha \in \widetilde{V_{\gamma}}$, a contradiction.

Note 2.14 The last two results together mean that for definably normal $X$, and any $\alpha \in \widetilde{X}$ there is a unique closed point $\rho(\alpha) \in \widetilde{X}$ which is a specialization of $\alpha$. i.e. there is a map $\rho: \widetilde{X} \longrightarrow X^{c}$, where $X^{c}$ is the subspace of closed points of $\widetilde{X}$.

Example 2.15 We note here that $X$ from Example 2.10 is not definably normal, explaining the lack of uniqueness of closed specialisations. Simply notice that the closed line segments $[\langle a-\epsilon, b\rangle,\langle a+\epsilon, b\rangle]$ and $[\langle a, b-\epsilon\rangle,\langle a, b+\epsilon\rangle]$ 
are disjoint closed definable subsets of $X$ but not separable by open definable sets.

If $\mathcal{N}$ is an o-minimal expansion of an ordered group, then by [vdd] Chapter VI, (3.5), every definable set is definably normal.

We obtain from Theorem 2.13 the following corollary.

Proposition 2.16 Let $X$ be a definably normal definable set. The subspace $X^{c}$ of closed points of $\widetilde{X}$ is a Hausdorff compact topological space and the mapping $\rho: \widetilde{X} \longrightarrow X^{c}$ is a continuous and closed retraction which sends every constructible subset of $\widetilde{X}$ into a closed subset of $X^{c}$.

Indeed, as shown in [cc] Proposition 3, the statement holds in any normal spectral space.

An important corollary of Theorem 2.13, is the following result which will play the role of paracompactness in o-minimal sheaf cohomology. The proof is similar to the one for constructible subsets of real spectra. See [br1], [D2], $[\mathrm{dk} 1]$.

Proposition 2.17 (The Shrinking Lemma) Let $X$ be a definably normal definable set. If $\left\{U_{i}: i=1, \ldots, n\right\}$ is a covering of $\widetilde{X}$ by open subsets of $\tilde{X}$, then there are constructible open subsets $V_{i}$ and constructible closed subsets $K_{i}$ of $\widetilde{X}(1 \leq i \leq n)$ with $V_{i} \subseteq K_{i} \subseteq U_{i}$ and $\widetilde{X}=\cup\left\{V_{i}: i=1, \ldots, n\right\}$.

Proof. We define open subsets $V_{i}$ and closed subsets $K_{i}$ of $\widetilde{X}(1 \leq i \leq n)$ by induction. Assume that the sets $V_{i}$ and $K_{i}$ are already constructed for $i=1, \ldots, m$ with $0 \leq m \leq n-1$ and have the following properties: (i) $V_{i} \subseteq K_{i} \subseteq U_{i}(1 \leq i \leq m)$; (ii) $V_{i}$ and $\widetilde{X} \backslash K_{i}$ are constructible $(1 \leq i \leq m)$ and (iii) $\left(\cup\left\{V_{i}: i=1, \ldots, m\right\}\right) \cup\left(\cup\left\{U_{i}: i=m+1, \ldots, n\right\}\right)=\tilde{X}$.

The sets $A=\widetilde{X} \backslash U_{m+1}$ and $B=\widetilde{X} \backslash\left[\left(\cup\left\{V_{i}: i=1, \ldots, m\right\}\right) \cup\left(\cup\left\{U_{i}\right.\right.\right.$ : $i=m+2, \ldots, n\})]$ are closed and disjoint subsets of $\widetilde{X}$. Hence, by Theorem 2.13 , there exist an open constructible neighbourhoods $W$ of $A$ and $V_{m+1}$ of $B$ with $W \cap V_{m+1}=\emptyset$. Define $K_{m+1}=\widetilde{X} \backslash W$. Then properties (i), (ii) and (iii) are fulfilled with $m$ replaced by $m+1$. Since $V_{i}$ and $\tilde{X} \backslash K_{i}$ are constructible open subsets of $\tilde{X}$, the sets $V_{i}$ and $K_{i}$ are constructible.

We note that this result could also be proved via the result [vdd] Chapter $\mathrm{VI},(3.6)$.

We end the section with the o-minimal spectrum of definable maps. The following is the o-minimal analogue of [BCR] Proposition 7.2.8, and is the standard model theoretic definition of the extension of a definable map to the type space. 
Definition 2.18 Let $f: X \longrightarrow Y$ be a definable map. Then there exists a unique mapping $\widetilde{f}: \widetilde{X} \longrightarrow \widetilde{Y}$, called the o-minimal spectrum of $f$, such that for $\alpha \in \widetilde{X}$ and for every definable subset $B$ of $Y$ we have $B \in \widetilde{f}(\alpha)$ if and only if $f^{-1}(B) \in \alpha$.

Remark 2.19 Let $f: X \longrightarrow Y$ be a definable map. Then the following hold:

- $\widetilde{f}^{-1}(\widetilde{B})=\widetilde{f^{-1}(B)}$ for every definable subset $B$ of $Y$.

- $\tilde{f}(\widetilde{A})=\widetilde{f(A)}$ for every definable subset $A$ of $X$.

- By Remark 2.3 (2), if the definable map $f: X \longrightarrow Y$ is continuous, then the mapping $\tilde{f}: \widetilde{X} \longrightarrow \widetilde{Y}$ is continuous.

The main property of the o-minimal spectrum of definable maps that we use, to get the base change theorem which is required for the Vietoris Begle theorem, is the following proposition.

Proposition 2.20 If $f: X \longrightarrow Y$ is a continuous definable map then for any $\alpha \in \widetilde{Y}$ we have that $\widetilde{f}^{-1}(\alpha)$ is quasi-compact.

Proof. $\quad \tilde{f}^{-1}(\alpha)=\bigcap\left\{\widehat{f^{-1}(B)}: B \in \alpha\right\}$, so $\tilde{f}^{-1}(\alpha)$ is compact in the constructible topology because each $\widehat{f^{-1}(B)}$ is (being the tilde of a definable set). Hence $\widetilde{f}^{-1}(\alpha)$ is quasi-compact in the spectral topology.

\section{Sheaves on definable sets}

Let DTOP be the category of definable sets and continuous definable maps. Definitions 2.2, 2.18 and Remark 2.19 give us the o-minimal tilde functor $\mathrm{DTOP} \longrightarrow \widehat{\mathrm{DTOP}}$, where $\widetilde{\mathrm{DTOP}}$ is the category whose objects are the o-minimal spectra of definable sets and the morphisms are the o-minimal spectra of continuous definable maps between definable sets.

Definition 3.1 Let $X$ be a definable set. The o-minimal site on $X$ consists of definable (relatively) open subsets of $X$, where the admissible coverings of a definable open $U$ are those coverings (by definable opens) with a finite subcovering. We denote by $\operatorname{Sh}_{\text {dtop }}(X)$ the category of sheaves of abelian groups on $X$ with respect to the o-minimal site on $X$. For the o-minimal spectrum $\tilde{X}$ of $X$, since it is a topological space, we use the classical notation $\operatorname{Sh}(\widetilde{X})$ to denote the category of sheaves of abelian groups on $\widetilde{X}$. 
Since the topology on the o-minimal spectrum $\widetilde{X}$ of $X$ is generated by the constructible open subsets, i.e., sets of the form $\widetilde{U}$ with $U$ an open definable subset of $X$, a sheaf on $\widetilde{X}$ is determined by its values on the sets $\widetilde{U}$ with $U$ an open definable subset of $X$. Thus, for a definable set $X$, we define the functors of the categories of sheaves of abelian groups

$$
\operatorname{Sh}_{\mathrm{dtop}}(X) \longrightarrow \operatorname{Sh}(\widetilde{X})
$$

which sends $\mathcal{F} \in \operatorname{Sh}_{\text {dtop }}(X)$ into $\widetilde{\mathcal{F}}$ where, for $U$ an open definable subset of $X$, we define $\widetilde{\mathcal{F}}(\widetilde{U})=\{\widetilde{s}: s \in \mathcal{F}(U)\} \simeq \mathcal{F}(U)$, and

$$
\operatorname{Sh}(\tilde{X}) \longrightarrow \operatorname{Sh}_{\mathrm{dtop}}(X)
$$

which sends $\widetilde{\mathcal{F}}$ into $\mathcal{F}$ where, for $U$ an open definable subset of $X$, we define $\mathcal{F}(U)=\{s: \widetilde{s} \in \widetilde{\mathcal{F}}(\widetilde{U})\} \simeq \widetilde{\mathcal{F}}(\widetilde{U})$.

Proposition 3.2 Let $X$ be a definable set. The functor $\operatorname{Sh}_{\mathrm{dtop}}(X) \longrightarrow$ $\operatorname{Sh}(\widetilde{X})$ is a well defined isomorphism of categories with inverse given by $\operatorname{Sh}(\tilde{X}) \longrightarrow \operatorname{Sh}_{\mathrm{dtop}}(X)$, hence $\operatorname{Sh}_{\mathrm{dtop}}(X)$ is an abelian category with enough injectives.

Proof. Let $\mathcal{F}$ be a sheaf in $\operatorname{Sh}_{\text {dtop }}(X), U$ an open definable subset of $X$ and suppose that $\left\{\widetilde{U}_{i}: i \in I\right\}$ is an (admissible) open cover of $\widetilde{U}$ in $\widetilde{X}$ and $\widetilde{s_{i}} \in \widetilde{\mathcal{F}}\left(\widetilde{U_{i}}\right)$ are sections such that $\widetilde{s_{i}}{\widetilde{U_{i}} \cap \widetilde{U_{j}}}={\widetilde{s_{j}}}_{\mid \widetilde{U_{i}} \cap \widetilde{U_{j}}}$. Since $\widetilde{U}$ is quasicompact, we may assume that $I$ is finite. Hence, $\left\{U_{i}: i \in I\right\}$ is an admissible open cover of $U$. But then, the sections $s_{i} \in \mathcal{F}\left(U_{i}\right)$ can be glued together to give a section $s \in \mathcal{F}(U)$. Consequently, the sections $\widetilde{s_{i}} \in \widetilde{\mathcal{F}}\left(\widetilde{U}_{i}\right)$ can be glued together to give a section $\widetilde{s} \in \widetilde{\mathcal{F}}(\widetilde{U})$.

Clearly, $\operatorname{Sh}(\widetilde{X}) \longrightarrow \operatorname{Sh}_{\text {dtop }}(X)$ is the inverse to $\operatorname{Sh}_{\text {dtop }}(X) \longrightarrow \operatorname{Sh}(\tilde{X})$.

Since the category of sheaves of abelian groups on a topological space is an abelian category with enough injectives (see [ks] Proposition 2.2.4 and 2.4.3 or [b] Chapter II, Theorem 3.2) and $\widetilde{X}$ is a topological space, it follows from the isomorphism $\operatorname{Sh}_{\mathrm{dtop}}(X) \longrightarrow \operatorname{Sh}(\tilde{X})$, that the same holds for $\operatorname{Sh}_{\mathrm{dtop}}(X)$.

Given a continuous definable map $f: X \longrightarrow Y$, we can define the direct image

$$
f_{*}: \operatorname{Sh}_{\mathrm{dtop}}(X) \longrightarrow \mathrm{Sh}_{\mathrm{dtop}}(Y)
$$

and the inverse image

$$
f^{*}: \operatorname{Sh}_{\mathrm{dtop}}(Y) \longrightarrow \operatorname{Sh}_{\mathrm{dtop}}(X)
$$


morphisms via the isomorphism of Proposition 3.2 from the direct image and inverse image morphisms in the category of sheaves of abelian groups in topological spaces treated in [b] Chapter I, Section 3 and 4:

$$
\widetilde{f_{*} \mathcal{F}}=\widetilde{f}_{*} \widetilde{\mathcal{F}} \text { and } \widetilde{f^{*} \mathcal{G}}=\widetilde{f^{*}} \widetilde{\mathcal{G}}
$$

for $\mathcal{F} \in \operatorname{Sh}_{\text {dtop }}(X)$ and $\mathcal{G} \in \operatorname{Sh}_{\text {dtop }}(Y)$.

The direct image and the inverse image are adjoint to each other

$$
\operatorname{Hom}\left(\mathcal{G}, f_{*} \mathcal{F}\right) \simeq \operatorname{Hom}\left(f^{*} \mathcal{G}, \mathcal{F}\right)
$$

and we have functoriality $\mathrm{id}_{*}=\mathrm{id},(f \circ g)_{*}=f_{*} \circ g_{*}, \mathrm{id}^{*}=\mathrm{id}$ and $(f \circ g)^{*}=$ $g^{*} \circ f^{*}$. Furthermore, from the fact that the inverse image and the direct image are adjoint, it follows that there are natural morphisms of functors $f^{*} \circ f_{*} \rightarrow$ id and id $\rightarrow f_{*} \circ f^{*}$ called the adjunction morphisms.

If $Z \subseteq X$ are definable sets, $j: Z \longrightarrow X$ is the inclusion and $\mathcal{F} \in$ $\operatorname{Sh}_{\text {dtop }}(X)$ the restriction is $\mathcal{F}_{\mid Z}=j^{*} \mathcal{F}$ and if $Z$ is closed, the extension by zero $\mathcal{F}_{Z}=j_{*} j^{*} \mathcal{F}$ is the sheaf such that the sequence $0 \longrightarrow \mathcal{F}_{Z}(U) \longrightarrow$ $\mathcal{F}(U) \longrightarrow \mathcal{F}(U \backslash Z) \longrightarrow 0$ is exact for every $U$ open definable subset of $X$. We also use the notation $\Gamma(U ; \mathcal{F})$ and $\Gamma_{Z}(U ; \mathcal{F})$ for $\mathcal{F}(U)$ and $\mathcal{F}_{Z}(U)$ respectively.

For details on all of the above see [ks] Chapter II, Section 2.3 or [b] Chapter I, Section 3 and 4.

Note that, using our shrinking lemma (Proposition 2.15) we can prove ominimal analogues of [D2] Lemmas 2.2, 2.3 and 2.4. Also we have in $\widetilde{\mathrm{DTOP}}$ o-minimal versions of the classical results [b] Chapter II, Proposition 5.3 and Theorem 5.4.

\section{O-minimal sheaf cohomology}

In this section we prove the existence of o-minimal sheaf cohomology satisfying the Eilenberg-Steenrod axioms adapted to the o-minimal site.

\subsection{O-minimal sheaf cohomology}

Let $X$ be a definable set and $\mathcal{F}$ a sheaf in $\operatorname{Sh}_{\text {dtop }}(X)$. We define the $o$-minimal sheaf cohomology groups by

$$
H^{n}(X ; \mathcal{F})=H^{n}(\widetilde{X} ; \widetilde{\mathcal{F}}) \text { for all } n \in \mathbb{N},
$$

where $H^{n}(\widetilde{X} ; \widetilde{\mathcal{F}})$ is classical sheaf cohomology (see $[\mathrm{ks}]$ or $[\mathrm{b}]$ ). 
If $f: X \longrightarrow Y$ is a continuous definable map, we define the induced homomorphism

$$
f^{*}: H^{*}(Y ; \mathcal{F}) \longrightarrow H^{*}\left(X ; f^{*} \mathcal{F}\right)
$$

in cohomology to be the same as the induced homomorphism

$$
\tilde{f}^{*}: H^{*}(\widetilde{Y} ; \widetilde{\mathcal{F}}) \longrightarrow H^{*}\left(\widetilde{X} ; \widetilde{f^{*}} \widetilde{\mathcal{F}}\right)
$$

in cohomology of the continuous map $\tilde{f}: \widetilde{X} \longrightarrow \widetilde{Y}$ of topological spaces. It follows from this definition and [b] Chapter II, 6.3 that the induced homomorphism $f^{*}: H^{*}(Y ; \mathcal{F}) \longrightarrow H^{*}\left(X ; f^{*} \mathcal{F}\right)$ in cohomology is, when we consider cohomology as a functor of sheaves in $\operatorname{Sh}_{\text {dtop }}(Y)$, a natural transformation of functors compatible with connecting homomorphisms.

If $X$ is a definable set, $A$ is a closed definable subset of $X$ and $\mathcal{F}$ a sheaf in $\operatorname{Sh}_{\mathrm{dtop}}(X)$, we define as above the relative o-minimal sheaf cohomology groups

$$
H^{n}(X, A ; \mathcal{F}) \text { for all } n \in \mathbb{N}
$$

by replacing $\mathcal{F}$ by $\mathcal{F}_{X \backslash A}$. Similarly, if $f:(X, A) \longrightarrow(Y, B)$ is a continuous definable map of closed pairs of definable sets (i.e., $A \subseteq X$ and $B \subseteq Y$ are closed definable subsets and $f: X \longrightarrow Y$ is a continuous definable map such that $f(A) \subseteq B$ ) and $\mathcal{F}$ a sheaf in $\operatorname{Sh}_{\text {dtop }}(Y)$, then the induced homomorphisms

$$
f^{*}: H^{*}(Y, B ; \mathcal{F}) \longrightarrow H^{*}\left(X, A ; f^{*} \mathcal{F}\right)
$$

in cohomology are defined as above by replacing $\mathcal{F}$ by $\mathcal{F}_{X \backslash B}$.

Note that by the isomorphism of Proposition 3.2, we could have defined the above o-minimal sheaf cohomology functor directly in the category of definable sets equipped with the o-minimal site. However, the approach we followed above is more convenient since we will often be able to refer directly to classical results in the category of topological spaces instead of having to prove them in the o-minimal context.

We have the following useful characterisation of the o-minimal cohomology groups.

Proposition 4.1 Let $X$ be a definably normal definable set and $\mathcal{F}$ a sheaf in $\operatorname{Sh}_{\text {dtop }}(X)$. Then for all $n \in \mathbb{N}$, the cohomology group $H^{n}(X ; \mathcal{F})$ is isomorphic to the $\breve{C e c h}$ cohomology group $\breve{H}^{n}(X ; \mathcal{F})$ relative to the o-minimal site on $X$, i.e., calculated using finite covers by open definable subsets of $X$.

This is the same as its semi-algebraic analogue in [cc] Proposition 5 and the proof is the same since one only uses that fact that $\widetilde{X}$ is a normal spectral 
space (Theorem 2.13). Similarly we have the following vanishing theorem which is the o-minimal version of $[\mathrm{cc}]$ Corollary 3. Here we use the fact that for a definable set $X$, we have $\operatorname{dim} X=\operatorname{dim}_{\mathrm{Krull}} \tilde{X}$, where $\operatorname{dim}_{\mathrm{Krull}} \tilde{X}$, the Krull dimension of $\widetilde{X}$, is the maximal length of proper specialisations of points in $\tilde{X}$. To see this use Lemma 2.11 and the fact that a cell of dimension $k$ is definably homeomorphic to an open cell in $N^{k}$ ([vdd] Chapter III, (2.7)).

Proposition 4.2 (Vanishing theorem) Let $X$ be a definably normal definable set and $\mathcal{F}$ a sheaf in $\operatorname{Sh}_{\text {dtop }}(X)$. Then

$$
H^{n}(X ; \mathcal{F})=0 \text { for all } n>\operatorname{dim} X \text {. }
$$

We have an o-minimal analogue of [D2] Theorem 3.1, from which, using our Proposition 2.8 we get an o-minimal base change theorem similar to [D2] Theorem 3.5. The Vietoris-Begle theorem follows from this o-minimal base change theorem using classical arguments (see, e.g. $[\mathrm{ks}]$ ). It is easy to see by compactness that a morphism $f: X \longrightarrow Y$ in $\widetilde{\mathrm{DTOP}}$ maps closed constructible subsets into closed sets if and only if $f$ is a closed map.

Theorem 4.3 (Vietoris-Begle theorem) Let $f: X \longrightarrow Y$ be a surjective morphism in $\widehat{\mathrm{DTOP}}$ that maps constructible closed subsets of $X$ onto closed subsets of $Y$. Let $\mathcal{F}$ be a sheaf in $\operatorname{Sh}(Y)$ and suppose that $Y$ is a subspace of a normal space in $\widehat{\mathrm{DTOP}}$. Assume that $f^{-1}(\beta)$ is connected and $H^{q}\left(f^{-1}(\beta) ; f^{*} \mathcal{F}_{\mid f^{-1}(\beta)}\right)=0$ for $q>0$ and all $\beta \in Y$. Then the induced map

$$
f^{*}: H^{*}(Y ; \mathcal{F}) \longrightarrow H^{*}\left(X ; f^{*} \mathcal{F}\right)
$$

is an isomorphism.

\subsection{The Eilenberg-Steenrod axioms}

Finally we are ready to prove the main result of the paper, namely that the o-minimal cohomology functor $H^{*}$ constructed above satisfies the o-minimal Eilenberg-Steenrod axioms:

Theorem 4.4 If $X$ is a definable set and $\mathcal{F}$ is a sheaf in $\operatorname{Sh}_{\mathrm{dtop}}(X)$, then the following hold:

Exactness Axiom. Let $A \subseteq X$ be a closed definable subset. If $i$ : $(A, \emptyset) \longrightarrow(X, \emptyset)$ and $j:(X, \emptyset) \longrightarrow(X, A)$ are the inclusions, then we have a natural exact sequence

$$
\cdots \longrightarrow H^{n}(X, A ; \mathcal{F}) \stackrel{j^{*}}{\rightarrow} H^{n}(X ; \mathcal{F}) \stackrel{i^{*}}{\rightarrow} H^{n}(A ; \mathcal{F}) \stackrel{d^{n}}{\rightarrow} H^{n+1}(X, A ; \mathcal{F}) \longrightarrow \cdots
$$


Excision Axiom. For every closed definable subset $A \subseteq X$ and definable open subset $U \subseteq X$ such that $U \subseteq A$, the inclusion $(X-U, A-U) \longrightarrow(X, A)$ induces isomorphisms

$$
H^{n}(X, A ; \mathcal{F}) \longrightarrow H^{n}(X-U, A-U ; \mathcal{F})
$$

for all $n \in \mathbb{N}$.

Homotopy Axiom. Let $[a, b] \subseteq N$ be a closed interval and $A \subseteq X$ a closed definable subset. Assume that $\mathcal{N}$ has definable Skolem functions, $X$ is definably normal and the projection $X \times[a, b] \longrightarrow X$ maps closed definable subsets of $X \times[a, b]$ onto closed definable subsets of $X$. If for $c \in[a, b]$,

$$
i_{c}:(X, A) \longrightarrow(X \times[a, b], A \times[a, b])
$$

is the continuous definable map given by $i_{c}(x)=(x, c)$ for all $x \in X$, then

$$
i_{a}^{*}=i_{b}^{*}: H^{n}\left(X \times[a, b], A \times[a, b] ; \pi^{*} \mathcal{F}\right) \longrightarrow H^{n}(X, A ; \mathcal{F})
$$

for all $n \in \mathbb{N}$.

Dimension Axiom. If $X$ is a one point set, then $H^{n}(X ; \mathcal{F})=0$ for all $n>0$ and $H^{0}(X ; \mathcal{F})=\mathcal{F}$.

Proof. Once we pass to $\widetilde{\mathrm{DTOP}}$ the proofs of the exactness and excision axioms are purely algebraic. See [b] Chapter II, Section 12, (22) and 12.8 respectively. The dimension axiom is also immediate.

The homotopy axiom will follow once we show that the projection map $\pi:(X \times[a, b], A \times[a, b]) \longrightarrow(X, A)$ induces an isomorphism

$$
\pi^{*}: H^{n}(X, A ; \mathcal{F}) \longrightarrow H^{n}\left(X \times[a, b], A \times[a, b] ; \pi^{*} \mathcal{F}\right)
$$

since by functoriality we obtain

$$
i_{a}^{*}=i_{b}^{*}=\left(\pi^{*}\right)^{-1}: H^{n}\left(X \times[a, b], A \times[a, b] ; \pi^{*} \mathcal{F}\right) \longrightarrow H^{n}(X, A ; \mathcal{F})
$$

for all $n \in \mathbb{N}$. By the exactness axiom it suffices to show that we have an isomorphism $\pi^{*}: H^{n}(X ; \mathcal{F}) \longrightarrow H^{n}\left(X \times[a, b] ; \pi^{*} \mathcal{F}\right)$. Equivalently we need to show that $\left.\tilde{\pi}^{*}: H^{n}(\widetilde{X} ; \widetilde{\mathcal{F}}) \longrightarrow H^{n}(\widetilde{X \times[a}, b] ; \tilde{\pi}^{*} \widetilde{\mathcal{F}}\right)$ is an isomorphism. For this we verify the hypothesis of the Vietoris-Begle theorem (Theorem 4.3).

By Theorem 2.13, $\widetilde{X}$ is normal. By the assumption, $\pi: X \times[a, b] \longrightarrow X$ maps closed definable subsets of $X \times[a, b]$ onto closed definable subsets of $X$. Therefore, $\tilde{\pi}: \widetilde{X \times[a, b]} \longrightarrow \tilde{X}$ maps constructible closed subset of $\widetilde{X \times[a, b]}$ onto (constructible) closed subsets of $\widetilde{X}$.

Since for each $\alpha \in \widetilde{X}, \widetilde{\pi}^{*} \widetilde{\mathcal{F}}_{\mid \widetilde{\pi}^{-1}(\alpha)}$ is the constant sheaf $\widetilde{\mathcal{F}}_{\alpha}$, it remains to show that $\widetilde{\pi}^{-1}(\alpha)$ is connected and acyclic i.e., $H^{q}\left(\widetilde{\pi}^{-1}(\alpha) ; F\right)=0$ for every $q>0$ and every abelian group $F$. 
Claim 4.5 Let $\alpha \in \widetilde{X}$ and let $\mathcal{N}^{\star}$ be the prime model of the first-order theory of $\mathcal{N}$ over $N \cup\{e\}$, where $e$ is an element realising the type $\alpha$. Then there exists a homeomorphism $t: \widetilde{\pi}^{-1}(\alpha) \longrightarrow \widetilde{[a, b]^{\star}}$, and so $\widetilde{\pi}^{-1}(\alpha)$ is quasicompact, connected and acyclic.

Define the map $t: \widetilde{\pi}^{-1}(\alpha) \longrightarrow \widetilde{[a, b]^{\star}}$ by sending $\gamma \in \widetilde{\pi}^{-1}(\alpha)$ to the type $t(\gamma)=\operatorname{tp}\left(c / N^{\star}\right)$ such that $(e, c)$ realises $\gamma$ in some saturated elementary extension of $\mathcal{N}^{\star}$. Since $\mathcal{N}$ has definable Skolem functions, every element in $N^{\star}$ is defined over $N \cup\{e\}$. Hence the map $t$ is a well defined. Indeed, suppose that $(e, c)$ and $(e, d)$ realise $\gamma$ in some saturated elementary extension of $\mathcal{N}^{\star}$ but $\operatorname{tp}\left(c / N^{\star}\right)$ is different from $\operatorname{tp}\left(d / N^{\star}\right)$. Then there is a first-order formula $\phi(u, m)$ with parameter $m \in N^{\star}$ such that $\phi(c, m)$ holds but $\phi(d, m)$ doesn't hold. As $m$ is defined over $N \cup\{e\}$, there exists a first-order formula $\psi(v, w)$ with parameters in $N$ such that $\psi(v, e)$ defines $m$. So $\exists v \psi(v, w) \wedge \phi(u, v)$ is realised by $(e, c)$ but not by $(e, d)$ which is a contradiction.

A similar argument shows that $t$ is injective. Let us show that $t$ is surjective. Let $\beta$ be a type in $\widetilde{[a, b]^{\star}}$. As above, every formula $\phi(u, m)$ in $\beta$ is equivalent to a formula of the form $\tau(e, u)$ where $\tau(w, u)$ is a formula over $N$. Clearly the type $\alpha$ is consistent with the collection $\Sigma(w, u)$ of all such formulas obtained from the formulas in $\beta$. Furthermore, $\alpha \cup \Sigma(w, u)$ determines a type $\gamma$ over $N$ such that $t(\gamma)=\beta$. In fact, let $\theta(w, u)$ be a first-order formula over $N$ and let $c$ be a realisation of $\beta$. Then either $\theta(e, c)$ holds in which case $\theta(e, u) \in \beta$ and $\theta(w, u) \in \Sigma(w, u)$ or $\theta(e, c)$ doesn't hold in which case $\neg \theta(e, u) \in \beta$ and $\neg \theta(w, u) \in \Sigma(w, u)$.

Noting that if $U$ is an open definable subset of $X \times[a, b]$, then $t(\widetilde{U} \cap$ $\left.\tilde{\pi}^{-1}(\alpha)\right)=\widetilde{r^{\star}\left(U^{\star}\right)}$ where $r: X \times[a, b] \longrightarrow[a, b]$ is the projection, it follows that $t$ is a open map. To show that $t$ is a homeomorphism, it remains to show that $t$ is continuous. Let $a<c_{1}<c_{2}<b$ be elements of $[a, b]^{\star}$ over $N^{\star}$. Since $c_{1}$ and $c_{2}$ are defined over $N \cup\{e\}$ (because $\mathcal{N}$ has definable Skolem functions), there are definable functions $f_{1}, f_{2}: A \subseteq X \longrightarrow[a, b]$ over $N$ such that $f_{i}(e)=c_{i}$ for $i=1,2$. As pointed out in the proof of [p] Proposition 2.2, the proof of [p] Proposition 2.1 shows that there exists an open definable subset $U$ of $X$ over $N$ containing $A$ and continuous definable functions $g_{1}, g_{2}: U \subseteq X \longrightarrow[a, b]$ over $N$ such that $g_{i \mid A}=f_{i}$ for $i=1,2$. But then $t\left(\widetilde{W} \cap \widetilde{\pi}^{-1}(\alpha)\right)=\widetilde{\left(c_{1}, c_{2}\right)}$ where $W=\left(g_{1}, g_{2}\right)_{U}$ is an open definable subset of $X \times[a, b]$ over $N$. Similarly, there are open definable subset $W_{i}(i=1,2)$ of $X \times[a, b]$ such that $t\left(\widetilde{W_{1}} \cap \widetilde{\pi}^{-1}(\alpha)\right)=\widetilde{\left[a, c_{1}\right)}$ and $t\left(\widetilde{W_{2}} \cap \widetilde{\pi}^{-1}(\alpha)\right)=\widetilde{\left(c_{2}, b\right]}$.

Since $t$ is a homeomorphism and $\widetilde{[a, b]^{\star}}$ is quasi-compact and connected (by Remark $2.3(3)), \widetilde{\pi}^{-1}(\alpha)$ is quasi-compact and connected. Furthermore, 
we have $H^{*}\left(\tilde{\pi}^{-1}(\alpha) ; F\right) \simeq H^{*}\left(\widetilde{[a, b]^{\star}} ; F\right) \simeq H^{*}\left([a, b]^{\star} ; F\right)$. By Proposition 4.1, $H^{*}\left([a, b]^{\star} ; F\right) \simeq \breve{H}^{*}\left([a, b]^{\star} ; F\right)$. Arguing as in [D2] page 124 , we conclude that $\breve{H}^{q}\left([a, b]^{\star} ; F\right)=0$ for all $q>0$ as required.

Let $f, g:(X, A) \longrightarrow(Y, B)$ be continuous definable maps with $A \subseteq X$ and $B \subseteq Y$ closed definable subsets and suppose that $s:(X \times[a, b], A \times$ $[a, b]) \longrightarrow(Y, B)$ is a definable homotopy between $f$ and $g$, meaning that $s$ is a continuous definable map such that $s \circ i_{a}=f$ and $s \circ i_{b}=g$. If $X$ satisfies the assumptions of the homotopy axiom and $F$ is a constant sheaf in $\operatorname{Sh}_{\text {dtop }}(X)$, then we get by functoriality

$$
f^{*}=g^{*}: H^{n}(Y, B ; F) \longrightarrow H^{n}(X, A ; F)
$$

for all $n \in \mathbb{N}$.

We can now prove the theorem from the introduction:

Proof. [of Theorem 1.1] Suppose that $\mathcal{N}$ is an o-minimal expansion of a group. By results in Chapter 6 of [vdd], every definable set is definably normal, $\mathcal{N}$ has definable Skolem functions and satisfies the additional assumption required for the homotopy axiom (Proposition 1.14 on page 97 of [vdd]). Now apply Theorem 4.4 to the constant sheaf $\mathbb{Z}$.

We end the section with the exactness for triples and the Mayer-Vietoris theorem.

Proposition 4.6 (Exactness for triples) Let $X$ be a definable set, $B \subseteq$ $A \subseteq X$ closed definable subsets and $\mathcal{F}$ a sheaf in $\operatorname{Sh}_{\text {dtop }}(X)$. Then there is an exact sequence for all $n \in \mathbb{N}$

$$
\rightarrow H^{n}(X, A ; \mathcal{F}) \rightarrow H^{n}(X, B ; \mathcal{F}) \rightarrow H^{n}(A, B ; \mathcal{F}) \rightarrow H^{n+1}(X, A ; \mathcal{F}) \rightarrow .
$$

In $\widetilde{\mathrm{DTOP}}$ the proof of Proposition 4.6 is as in [b] Chapter II, Section 12, (24). If $X$ is a definable set and $B \subseteq A \subseteq X$ are closed definable subsets, then by the excision axiom, $(X ; A, B)$ is an excisive triad meaning that the inclusion $(A, A \cap B) \longrightarrow(A \cup B, B)$ map induces isomorphisms

$$
H^{*}(A \cup B, B ; \mathcal{F}) \simeq H^{*}(A, A \cap B ; \mathcal{F})
$$

for every sheaf $\mathcal{F}$ in $\operatorname{Sh}_{\text {dtop }}(X)$. Thus, by going to $\widetilde{\text { DTOP }}$, the following holds (see [b] Chapter II, Section 13, (32)).

Proposition 4.7 (Mayer-Vietoris) Let $X$ and $Z$ be definable sets and let $X_{1}, X_{2}$ and $Z_{1}, Z_{2}$ be closed definable subsets such that $X=X_{1} \cup X_{2}$ and 
$Z=Z_{1} \cup Z_{2}$. Let $\mathcal{F}$ be a sheaf in $\operatorname{Sh}_{\mathrm{dtop}}(X)$. Assume that we have the following commutative diagram of inclusions

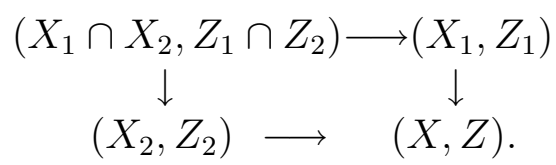

Then there is an exact sequence for all $n \in \mathbb{N}$

$$
\begin{aligned}
& \cdots \rightarrow H^{n}(X, Z ; \mathcal{F}) \rightarrow H^{n}\left(X_{1}, Z_{1} ; \mathcal{F}\right) \oplus H^{n}\left(X_{2}, Z_{2} ; \mathcal{F}\right) \rightarrow \\
& \quad \rightarrow H^{n}\left(X_{1} \cap X_{2}, Z_{1} \cap Z_{2} ; \mathcal{F}\right) \rightarrow H^{n+1}(X, Z ; \mathcal{F}) \rightarrow \cdots
\end{aligned}
$$

\section{References}

[bs] J.Bell and A.Slomson Models and ultraproducts: An introduction North Holland 1969.

[BCR] J.Bochnak, M. Coste and M-F. Roy Real Algebraic Geometry Springer-Verlag 1998.

[b] G.Bredon Sheaf theory Second Edition Springer-Verlag 1997.

[br1] G.W.Brumfiel Witt rings and K-theory Rocky Mountain J. Math. 14 (4) (1984) 733-765.

[c] M. Coste An introduction to o-minimal geometry Dip. Mat. Univ. Pisa, Dottorato di Ricerca in Matematica, Istituti Editoriali e Poligrafici Internazionali, Pisa (2000). Available in RAAG preprint server 2000 (http://ihp-raag.org/).

[cc] M.Carral and M.Coste Normal spectral spaces and their dimensions J. Pure and Appl. Algebra 30 (3) (1983) 227-235.

[cr] M.Coste and M.F.Roy La topologie du spectre réel in Ordered fields and real algebraic geometry, Contemporary Mathematics 8 (1982) 27-59.

[D2] H.Delfs The homotopy axiom in semi-algebraic sheaf cohomology J. reine angew. Maths. 355 (1985) 108-128.

[dk1] H.Delfs and M.Knebusch Separation, retraction and homotopy extension in semi-algebraic spaces Pacific J. Math. 114 (1984) 47-71. 
[vdd] L. van den Dries Tame topology and o-minimal structures Cambridge University Press 1998.

[eo] M. Edmundo and M. Otero Definably compact abelian groups. J. Math. Log. 4 (2) (2004) 163-180.

[h] M.Hochster Prime ideal structure in commutative rings Trans. Amer. Math. Soc. 142 (1969) 43-60.

[hpp] E. Hrushovski, Y. Peterzil and A. Pillay Groups, Measures, and the NIP, ArXiv.org math.LO/060744.

[ks] M.Kashiwara and P.Shapira Sheaves on manifolds Springer Verlag 1990.

[p] A.Pillay Sheaves of continuous definable functions J. Symbolic Logic 53 (4) (1988) 1165-1169.

[Wo] A.Woerheide O-minimal homology PhD. Thesis (1996), University of Illinois at Urbana-Champaign. 